\title{
SM-GR Reconciliation and Dark Sector
}

\author{
Durmuş Demir \\ Faculty of Engineering and Natural Sciences, Sabancl University, 34956 Tuzla, İstanbul, Turkey
}

\begin{abstract}
Poincare breaking scale causes explicit gauge invariance breaking at the loop level in the standard model (SM) - a renormalizable QFT in flat spacetime. In this talk, we show that gauge invariance can be restored by extending the general covariance by a covariance relation for curvature such that this extended covariance carries effective QFTs into curved spacetime to lead up to QFT-GR reconciliation, with renormalized QFTs and emergent GR. This mechanism predicts the existence of new physics beyond the SM (BSM), and does not necessitate the BSM sector to have any non-gravitational coupling with the SM. The BSM sector can have a dark subsector comprising the dark matter, dark energy, and other possible SM-singlet fields.
\end{abstract}

Keywords: UV cutoff, emergent GR, BSM sector, dark sector

DOI: 10.31526/ACP.NDM-2020.2

\section{INTRODUCTION}

The GR-QFT reconciliation is a long-standing problem. It is well known that classical field theories [1], governed by actions $S_{c l}(\eta, \psi, \partial \psi)$ of the fields $\psi$ in the flat spacetime of metric $\eta_{\mu \nu}$, are carried into curved spacetime of a metric $g_{\mu \nu}$ by letting

$$
S_{c l}(\eta, \psi, \partial \psi) \hookrightarrow S_{c l}(g, \psi, \nabla \psi)+\text { "curvature sector" }
$$

in accordance with general covariance [2], which is expressed by the map

$$
\eta_{\mu \nu} \hookrightarrow g_{\mu \nu}, \partial_{\mu} \hookrightarrow \nabla_{\mu}
$$

such that the Levi-Civita connection

$$
{ }^{g} \Gamma_{\mu \nu}^{\lambda}=\frac{1}{2} g^{\lambda \rho}\left(\partial_{\mu} g_{v \rho}+\partial_{\nu} g_{\rho \mu}-\partial_{\rho} g_{\mu \nu}\right)
$$

sets the covariant derivative $\nabla_{\mu}$, the Ricci curvature $R_{\mu v}(g \Gamma)$, and the scalar curvature $R(g)=g^{\mu v} R_{\mu \nu}\left({ }^{g} \Gamma\right)$. The "curvature sector" in (1), added by hand for $g_{\mu \nu}$ to be able to gain dynamics, must be of the form

$$
\text { "curvature sector" }=\int d^{4} x \sqrt{-g}\left\{-\frac{\tilde{M}^{2}}{2} R(g)+\tilde{c}_{2} R(g)^{2}+\frac{\tilde{c}_{3}}{\tilde{M}^{2}} R(g)^{3}+\ldots\right\}
$$

if it is to lead to GR. This procedure makes it clear that general covariance can carry classical field theories into curved spacetime if the curvature sector is structured judiciously [3].

Can general covariance carry also QFTs into curved spacetime? The answer is no. The reason is that QFTs are specific to flat affine spacetime $[4,5,6]$, and cannot therefore exist in curved spacetime in which Poincare invariance, wave-particle duality, and vacuum uniqueness are all lost [7]. This is a deadlock but, plausibly, an unlockable deadlock in that the uncharted land of effective QFTs may provide a way out. Indeed, effective QFTs resemble classical field theories in view of their long-wavelength field content with loop-corrected couplings, and their transformation into curved spacetime may reveal certain clues about possible QFT-GR concord.

In the rest, it is all effective QFTs. In what follows analyses and discussions will be given for a generic QFT to explicate the generality of the mechanisms to be constructed. The examples, estimates and predictions will, however, be based on the SM - the experimentally confirmed model of nature at the Fermi energies. In this regard, Sec. 2 reveals the nature of the UV cutoff, Sec. 3 the effects of the UV cutoff on gauge invariance, Sec. 4 the restoration of gauge invariance by curvature, and Sec. 5 the sought QFT-GR reconciliation. Sec. 6 concludes.

\section{POINCARE BREAKING SCALE AS THE UV CUTOFF}

The effective QFTs take shape with a UV cutoff. The problem is to determine that UV cutoff. To do that, it proves useful to distinguish between two kinds of mass scales: 
1. The first kind refers to the masses $m_{i}$ of the quantum fields $\psi_{i}$. They are propagator poles. They are Casimir invariants of the Poincare group [8]. They respect Poincare invariance. Thus, none of them, not a single $m_{i}$, can carve out a cutoff. The cutoff must be an outsider to QFTs. (This does not mean that QFTs cannot be analyzed using cutoff regularization [9].)

2. The second kind refers to curvature. It is an outsider to flat spacetime QFTs but it has a close relative there: a physical cutoff $\Lambda_{\wp}$. They are relatives because they both break Poincare invariance. In fact, $\Lambda_{\wp}$ can be visualized as shadow of curvature on flat spacetime so that incorporation of gravity into QFTs can be construed as reconstruction of curvature from its shadow. The Poincare breaking scale $\Lambda_{\wp}$ must lie above all the $m_{i}$ for entire QFT dynamics to be contained. Then, as the UV cutoff, $\Lambda_{\wp}$ restricts loop momenta $\ell_{\mu}$ into the finite interval

$$
-\Lambda_{\wp}^{2} \leq \ell_{\mu} \ell^{\mu} \leq \Lambda_{\wp}^{2}
$$

and renders thus all loop corrections finite and physical. Its affinity to curvature ensures that $\Lambda_{\wp}$ is physical, unique, and reduces always realistic QFTs to effective QFTs [10].

This correspondence between the Poincare breaking scale (UV cutoff) in flat spacetime and the curvature in curved spacetime eventuates in a covariance relation with which QFTs can be carried into curved spacetime in a way consistent with gravitational and field-theoretic structures [11].

\section{GAUGE SYMMETRY BREAKING BY THE UV CUTOFF}

The matter loops, whose momenta range in the band (5), induce quantum corrections to all the masses, couplings and fields in QFTs. Their effects on relevant operators are particularly important in view mainly of their strong UV sensitivities. To see how, it suffices to comparatively analyze the field masses and the vacuum energy:

1. The pure loop-induced masses

$$
\delta M_{V}^{2}=c_{V} \Lambda_{\wp}^{2}
$$

for massless gauge bosons $V_{\mu}$ involve $\Lambda_{\wp}$ and only $\Lambda_{\wp}$. They explicitly break gauge symmetries as exemplified in Table 1 with the SM gauge bosons. This means that the UV cutoff $\Lambda_{\wp}$, conjured up as a mark of curvature on flat spacetime, gives cause to explicit color and charge breaking (CCB) [12] at the loop level (see [13] for spontaneous CCB).

2. The fermion mass corrections

$$
\delta m_{f}=m_{f} \sum_{i} \hat{c}_{f \psi_{i}} \log \frac{m_{i}^{2}}{\Lambda_{\wp}^{2}}
$$

involve only the logarithmic ratio of the two scales. This means that the fermion sector maintains gauge invariance and remains insensitive to the UV effects.

3. The corrections to scalar masses

$$
\delta m_{\phi}^{2}=c_{\phi} \Lambda_{\wp}^{2}+\sum_{i} \hat{c}_{\phi \psi_{i}} m_{i}^{2} \log \frac{m_{i}^{2}}{\Lambda_{\wp}^{2}}
$$

involve both $\Lambda_{\wp}^{2}$ and $m_{i}^{2}$. The sheer $\Lambda_{\wp}^{2}$ contribution, whose loop factor $c_{\phi}$ is given in Table 1 for the SM Higgs boson, gives cause to the big hierarchy problem [14].

4. Finally, the shift in the vacuum energy

$$
\delta V=c_{\varnothing} \Lambda_{\wp}^{4}+\sum_{i} c_{\psi_{i}} m_{i}^{2} \Lambda_{\wp}^{2}+\sum_{i} \hat{c}_{\psi_{i}} m_{i}^{4} \log \frac{m_{i}^{2}}{\Lambda_{\wp}^{2}}
$$

involves quartics and quadratics of both $\Lambda_{\wp}$ and $m_{i}$. It is exemplified in Table 1 for the SM vacuum energy. It gathers both scales marginally, with of course no physical effects in flat spacetime $[15,16]$.

The loop corrections (6), (7), (8) and (9) form a quantum effective action $S\left(\eta, \psi, \Lambda_{\wp}\right)$ describing the effective QFT below $\Lambda_{\wp}$. If this effective QFT acts like classical field theories then the general covariance map in (2) must be able to carry it into curved spacetime as

$$
S\left(\eta, \psi, \Lambda_{\wp}\right) \hookrightarrow S\left(g, \psi, \Lambda_{\wp}\right)+\int d^{4} x \sqrt{-g}\left\{-\frac{\tilde{M}^{2}}{2} R(g)+\tilde{c}_{2} R(g)^{2}+\frac{\tilde{c}_{3}}{\tilde{M}^{2}} R(g)^{3}+\ldots\right\}
$$

with the curvature sector in (4). The problem with this action is that $\tilde{M}, \tilde{c}_{2}, \tilde{c}_{3}, \cdots$ are all incalculable constants [17, 11]. The reason is that matter loops have been used up already in forming the flat spacetime effective action $S\left(\eta, \psi, \Lambda_{\wp}\right)$, and there have remained thus no loops to induce any extra interaction, with or without curvature. This incalculability problem, which reveals the difference between classical and effective field theories, ensures that curvature sector in effective QFTs cannot be added by hand with arbitrary constants. 
Table 1: The loop factors $c_{V}$ at one loop and various problems they give cause for.

\begin{tabular}{l|l|l|l}
\hline Loop Factor & SM Fields & SM Value & Problems Caused \\
\hline \hline$c_{V}$ & gluon & $\frac{21 g^{2}}{16 \pi^{2}}$ & color breaking \\
\hline$c_{V}$ & weak gauge bosons & $\frac{21 g_{2}^{2}}{16 \pi^{2}}$ & isospin breaking \\
\hline$c_{V}$ & hypercharge gauge boson & $\frac{39 g_{1}^{2}}{32 \pi^{2}}$ & hypercharge breaking \\
\hline$c_{\phi}$ & Higgs boson $h$ & $\frac{g_{2}^{2} \operatorname{str}\left[m^{2}\right]}{8 \pi^{2} M_{W}^{2}} \approx-\frac{g_{2}^{2} m_{t}^{2}}{\pi^{2} M_{W}^{2}}$ & big hierarchy problem \\
\hline$c_{\varnothing}=-\frac{\operatorname{str}[1]}{128 \pi^{2}}$ & over all the SM fields & $\frac{31}{32 \pi^{2}}$ & none (flat spacetime) \\
\hline$\sum_{i} c_{\psi_{i}} m_{i}^{2}=\frac{\operatorname{str}\left[m^{2}\right]}{32 \pi^{2}}$ & over all the SM fields & $\approx-\frac{m_{t}^{2}}{4 \pi^{2}}$ & none (flat spacetime) \\
\hline
\end{tabular}

\section{GAUGE SYMMETRY RESTORATION BY CURVATURE}

The main implication of the incalculability problem is that curvature must arise from within $S\left(g, \psi, \Lambda_{\wp}\right)$ in order not to hinge on arbitrary, incalculable constants. This can be taken to imply that there must exist some covariance relation between the mass scales in $S\left(g, \psi, \Lambda_{\wp}\right)$ and the curvature. To determine if such a relation exists, it proves efficacious to focus on the gauge sector first, wherein the loop-induced masses in (6) set the effective action

$$
\delta S_{V}\left(\eta, \Lambda_{\wp}\right)=\int d^{4} x \sqrt{-\eta} c_{V} \Lambda_{\wp}^{2} \operatorname{Tr}\left[\eta_{\mu \nu} V^{\mu} V^{\nu}\right]
$$

as part of the total effective QFT action $S\left(\eta, \psi, \Lambda_{\wp}\right)$. This action breaks gauge symmetries explicitly. It does so in curved spacetime, too, if carried there through the incalculability-barred map in (10). This conundrum leads to a pivotal question: Is it possible to carry (11) into curved spacetime in a way restoring gauge symmetries? Can gauge invariance be the basis for incorporating gravity into effective QFTs? The answer is "yes". To see how, it proves useful to start with the self-evident identity [17, 11]

$$
\delta S_{V}\left(\eta, \Lambda_{\wp}^{2}\right)=\delta S_{V}\left(\eta, \Lambda_{\wp}^{2}\right)-I_{V}(\eta)+I_{V}(\eta)
$$

in which the gauge-invariant kinetic construct

$$
I_{V}(\eta)=\int d^{4} x \sqrt{-\eta} \frac{c_{V}}{2} \operatorname{Tr}\left\{\eta_{\mu \alpha} \eta_{\nu \beta} V^{\mu \nu} V^{\alpha \beta}\right\}
$$

is subtracted from and added back to $\delta S_{V}\left(\eta, \Lambda_{\wp}^{2}\right)$. This construct, involving the loop factor $c_{V}$ and the field strength tensor $V_{\mu \nu}$, leads to the expanded gauge boson mass action

$$
\delta S_{V}\left(\eta, \Lambda_{\wp}^{2}\right)=-I_{V}(\eta)+\int d^{4} x \sqrt{-\eta} c_{V} \operatorname{Tr}\left\{V^{\mu}\left(-D_{\mu \nu}^{2}+\Lambda_{\wp}^{2} \eta_{\mu \nu}\right) V^{\nu}+\partial_{\mu}\left(\eta_{\alpha \beta} V^{\alpha} V^{\beta \mu}\right)\right\}
$$

if, at the right hand side of (12), $\delta S_{V}$ is replaced with (11), " $-I_{V}$ " is left untouched, and yet " $+I_{V}$ " is integrated by-parts to contain $D_{\mu \nu}^{2}=D^{2} \eta_{\mu \nu}-D_{\mu} D_{v}-V_{\mu \nu}$, where $D_{\mu}$ is gauge-covariant derivative. Then, the reformed effective action $\delta S_{V}\left(\eta, \Lambda_{\wp}^{2}\right)$ in (14) changes to

$$
\delta S_{V}\left(g, \Lambda_{\wp}^{2}\right)=-I_{V}(g)+\int d^{4} x \sqrt{-g} C_{V} \operatorname{Tr}\left\{V^{\mu}\left(-\mathcal{D}_{\mu \nu}^{2}+\Lambda_{\wp}^{2} g_{\mu \nu}\right) V^{\nu}+\nabla_{\mu}\left(g_{\alpha \beta} V^{\alpha} V^{\beta \mu}\right)\right\}
$$

under the general covariance map (2) such that $\mathcal{D}_{\mu}$ is the gauge-covariant derivative with respect to $\nabla_{\mu}$, and $\mathcal{D}_{\mu \nu}^{2}=\mathcal{D}^{2} g_{\mu \nu}-$ $\mathcal{D}_{\mu} \mathcal{D}_{\nu}-V_{\mu \nu}$.

Here, a short glance at (15) reveals that $\delta S_{V}\left(g, \Lambda_{\wp}^{2}\right)$ would vanish identically if $\Lambda_{\wp}^{2} g_{\mu \nu}$ were replaced with $R_{\mu v}\left({ }^{g} \Gamma\right)$ because

$$
\int d^{4} x \sqrt{-g} c_{V} \operatorname{Tr}\left\{V^{\mu}\left(-\mathcal{D}_{\mu \nu}^{2}+R_{\mu \nu}\left({ }^{g} \Gamma\right)\right) V^{v}+\nabla_{\mu}\left(g_{\alpha \beta} V^{\alpha} V^{\beta \mu}\right)\right\}=I_{V}(g)
$$

as a clear result. This pivotal feature is, however, highly problematic since $\Lambda_{\wp}^{2} g_{\mu \nu} \hookrightarrow R_{\mu v}\left({ }^{g} \Gamma\right)$ contradicts with $\eta_{\mu \nu} \hookrightarrow g_{\mu \nu}$. If it were not for this contradiction, metamorphosis of curvature from $\Lambda_{\wp}^{2} g_{\mu v}$ would provide a perfect solution to the CCB [17, 11]. This contradiction is an impasse but it can be overcome by considering a more general map

$$
\Lambda_{\wp}^{2} g_{\mu \nu} \hookrightarrow \mathbb{R}_{\mu \nu}(\Gamma)
$$


in which $\mathbb{R}_{\mu \nu}(\Gamma)$ is the Ricci curvature of a symmetric affine connection $\Gamma_{\mu \nu}^{\lambda}$ (bearing no a priori relationship to the Levi-Civita connection $8 \Gamma_{\mu \nu}^{\lambda}$ ) [18]. The metamorphosis of $\Lambda_{\wp}^{2} g_{\mu \nu}$ into $\mathbb{R}_{\mu \nu}(\Gamma)$ goes parallel with the metamorphosis of $\eta_{\mu \nu}$ into $g_{\mu \nu}$ as a correspondence between physical quantities in flat and curved spacetimes, and removes the contradiction since the two maps, (2) and (17), involve independent dynamical variables. In fact, affine curvature may well be the missing substance in general covariance [3]. In consequence, the action (15) gets recast into a completely new form

$$
\delta S_{V}(g, \mathbb{R})=-I(g, V)+\int d^{4} x \sqrt{-g} c_{V} \operatorname{Tr}\left\{V^{\mu}\left(-\mathcal{D}_{\mu \nu}^{2}+\mathbb{R}_{\mu \nu}(\Gamma)\right) V^{\nu}+\nabla_{\mu}\left(g_{\alpha \beta} V^{\alpha} V^{\beta \mu}\right)\right\}
$$

under the maps (2) and (17), and reduces to

$$
\left.\delta S_{V}(g, \mathbb{R}, R)=\int d^{4} x \sqrt{-g} c_{V} \operatorname{Tr}\left\{V^{\mu}\left(\mathbb{R}_{\mu \nu}(\Gamma)-R_{\mu \nu}(g) \Gamma\right)\right) V^{\nu}\right\}
$$

through the identity (16). But, this reduced action is non-vanishing. Namely, the CCB [12] continues to catabolize the QFTs.

\section{QFT-GR RECONCILIATION}

It is clear that the CCB action $\delta S_{V}(g, \mathbb{R}, R)$ in (19) is suppressed only if $\mathbb{R}_{\mu v}(\Gamma)$ falls in close vicinity of $R_{\mu v}(g \Gamma)$. And the question of if such a suppression regime is ever attainable is decided by the dynamics of $\Gamma_{\mu \nu}^{\lambda}$, which is derived below systematically by highlighting important points and revealing their physics implications.

The first important point revolves around the identity (16) with which the action (18) reduces to (19). It rests implicitly on the condition that $c_{V}$ remains unchanged under (17). This means that $\log \Lambda_{\wp}$, which arises in $c_{V}$ at higher loops, must remain untouched while $\Lambda_{\wp}^{2}$ itself morphs into curvature as in (17). This condition, which might seem artificial at first sight, is actually what is needed for the renormalization of QFTs in course of the incorporation of gravity [20, 10, 11]. Indeed, logarithmic parts of (7), (8) and (9) lead to the effective action (to be completed by including the trilinear and quadrilinear corrections)

$$
\delta \hat{S}\left(g, \psi, \log \Lambda_{\wp}\right) \supset-\sum_{i} \hat{c}_{\psi_{i}} m_{i}^{4} \log \frac{m_{i}^{2}}{\Lambda_{\wp}^{2}}-\sum_{i, \phi} \hat{c}_{\phi \psi_{i}} m_{i}^{2} \log \frac{m_{i}^{2}}{\Lambda_{\wp}^{2}} \phi^{\dagger} \phi-\sum_{i, f} \hat{c}_{f \psi_{i}} \log \frac{m_{i}^{2}}{\Lambda_{\wp}^{2}} m_{f} \bar{f} f
$$

so that the improved action

$$
S_{\mathrm{QFT}}\left(g, \psi, \log \Lambda_{\wp}\right)=S_{c l}(g, \psi)+\delta \hat{S}\left(g, \psi, \log \Lambda_{\wp}\right)
$$

is of the same form as $S_{c l}(g, \psi)$ except that all of its fields and couplings get regularized with $\log \Lambda_{\wp}$ corrections. Needless to say, the formal equivalence [21]

$$
\log \Lambda_{\wp}^{2}=\frac{1}{\epsilon}-\gamma_{E}+1+\log 4 \pi \mu^{2}
$$

translates $S_{\mathrm{QFT}}\left(g, \psi, \log \Lambda_{\wp}\right)$ into dimensional regularization in $4+\epsilon$ dimensions such that the discard of $1 / \epsilon$ pieces results in the MS renormalization of QFTs at the scale $\mu$. In general, $\mu$-independence of scattering amplitudes leads to the renormalization group equations.

The second important point concerns $\Lambda_{\wp}^{2}$ and $\Lambda_{\wp}^{4}$ terms in (6), (8) and (9). In fact, the flat spacetime effective action corresponding to (8) plus (9)

$$
\delta S_{\varnothing \phi}\left(\eta, \Lambda_{\wp}\right)=\int d^{4} x \sqrt{-\eta}\left\{-c_{\varnothing} \Lambda_{\wp}^{4}-\sum_{i} c_{\psi_{i}} m_{i}^{2} \Lambda_{\wp}^{2}-c_{\phi} \phi^{\dagger} \phi \Lambda_{\wp}^{2}\right\}
$$

combines, after mapping through (2) and (17), with the action (19) corresponding to (6) and leads to a curvature sector which is not the incalculable one in (4) but a completely determined one

$$
\text { "curvature sector" }=\int d^{4} x \sqrt{-g}\left\{-Q^{\mu v} \mathbb{R}_{\mu \nu}(\Gamma)+\frac{1}{16} c_{\varnothing}\left(g^{\mu v} \mathbb{R}_{\mu v}(\Gamma)\right)^{2}-c_{V} R_{\mu \nu}\left({ }^{g} \Gamma\right) \operatorname{Tr}\left\{V^{\mu} V^{v}\right\}\right\}
$$

in which the disformal metric

$$
Q_{\mu \nu}=\left(\frac{1}{4} \sum_{i} c_{\psi_{i}} m_{i}^{2}+\frac{1}{4} c_{\phi} \phi^{\dagger} \phi+\frac{1}{8} c_{\varnothing} g^{\alpha \beta} \mathbb{R}_{\alpha \beta}(\Gamma)\right) g_{\mu \nu}-c_{V} \operatorname{Tr}\left\{V_{\mu} V_{v}\right\}
$$


involves all the scalars $\phi$ and vectors $V_{\mu}$, with implied summation.

The third important point refers to the curvature sector in (24), which ensures that the fundamental scale of gravity

$$
M_{P l}^{2}=\frac{1}{2} \sum_{i} c_{\psi_{i}} m_{i}^{2}
$$

arises as a pure quantum effect, and its one-loop value $\left(M_{P l}^{2}\right)_{1-\text { loop }}=\operatorname{str}\left\{m^{2}\right\} / 64 \pi^{2}$ reveals that the bosonic sector of QFTs must outweigh (some 1000 Planckian bosons or many more sub-Planckian bosons, with a relatively light fermion sector). It thus turns out that the QFTs themselves set the scale of gravity. In the SM, $M_{P l}^{2}$ in (26) comes out wrong both in sign and size, as revealed by the last row of Table 1 . This means that the SM needs be extended by some BSM sector so that $M_{P l}$ can come out right via the BSM mass spectrum $[10,11]$. The end result is that the SM cannot be the whole story; there must exist a BSM sector, which, according to (26), does not have interact with the SM non-gravitationally. Needless to say, the BSM sector can form (fully or partially) a dark sector $[11,22]$ containing the dark matter, dark energy and other possible non-SM phenomena.

The fourth important is about the equation of motion for $\Gamma_{\mu v}^{\lambda}$, which is stipulated by the stationarity of the action (24) against variations in $\Gamma_{\mu \nu}^{\lambda}$. It takes the compact form

$$
{ }^{\Gamma} \nabla_{\lambda} Q_{\mu \nu}=0
$$

and its solution $[18,19]$

$$
\Gamma_{\mu \nu}^{\lambda}={ }^{g} \Gamma_{\mu \nu}^{\lambda}+\frac{1}{2}\left(Q^{-1}\right)^{\lambda \rho}\left(\nabla_{\mu} Q_{\nu \rho}+\nabla_{\nu} Q_{\rho \mu}-\nabla_{\rho} Q_{\mu \nu}\right)
$$

falls within the close proximity of ${ }^{g} \Gamma_{\mu \nu}^{\lambda}$ due to the enormity of $M_{P l}$. In fact, the affine curvature expands as

$$
\mathbb{R}_{\mu \nu}(\Gamma)=R_{\mu \nu}\left({ }^{g} \Gamma\right)+\frac{1}{M_{P l}^{2}}\left(\nabla^{\alpha} \nabla_{\mu} \delta_{v}^{\beta}+\nabla^{\alpha} \nabla_{\nu} \delta_{\mu}^{\beta}-\square \delta_{\mu}^{\alpha} \delta_{v}^{\beta}-\nabla_{v} \nabla_{\mu} g^{\alpha \beta}\right) Q_{\alpha \beta}+\mathcal{O}\left(M_{P l}^{-4}\right)
$$

with a remainder which invariably involves derivatives (not masses) of $\phi$ and $V_{\mu}$ [11].

The fifth important point pertains to the CCB action in (19). It undergoes a strong suppression

$$
\int d^{4} x \sqrt{-g} \sum_{V} c_{V} \operatorname{Tr}\left\{V^{\mu}\left(\mathbb{R}_{\mu v}(\Gamma)-R_{\mu \nu}\left({ }^{g} \Gamma\right)\right) V^{\nu}\right\}=\int d^{4} x \sqrt{-g}\left\{0+\mathcal{O}\left(M_{P l}^{-2}\right)\right\}
$$

under the solution of the affine curvature in (29). The CCB is thus prevented modulo $\mathcal{O}\left(M_{P l}^{-2}\right)$ effects [11]. It turns out that extension of general covariance (2) by the curvature map (17) does indeed restore gauge symmetries (listed in Table 1 for the SM) through the identity (12).

The sixth important point relates to the metric-affine curvature sector in (24) [18, 19, 23], which gets to the GR realm

$$
\text { "curvature sector" }=\int d^{4} x \sqrt{-g}\left\{-\frac{1}{2} M_{P l}^{2} R(g)-\frac{1}{4} c_{\phi} \phi^{\dagger} \phi R(g)-\frac{1}{16} c_{\varnothing} R(g)^{2}+\mathcal{O}\left(M_{P l}^{-2}\right)\right\}
$$

under the affine curvature in (29). This action reveals that the scalar masses (8) cannot give rise to the big hierarchy problem [14] in Table 1 as their $\Lambda_{\wp}^{2}$ parts eventuate in the non-minimal coupling $c_{\phi} / 4$ between the scalars and the curvature scalar [24]. By the same token, the vacuum energy in (9) cannot give cause to the cosmological constant problem [15] in Table 1 as its $\Lambda_{\wp}^{2}\left(\Lambda_{\wp}^{4}\right)$ part leads to the Einstein-Hilbert (quadratic curvature [25]) term. (The quadratic term can enable the Planck-favored Starobinsky inflation $[26,27]$ for $\operatorname{str}[1] \sim 10^{9}$, as implied by Table 1.) The problems in Table 1 are not the whole story, however. Indeed, as revealed by the logarithmic action in (20), heavy fields $\Psi$ destabilize light scalars $\phi$ unless they couple with seesawic strength $m_{\phi}^{2} / m_{\Psi}^{2}$ $[10,11,28]$. Likewise, the logarithmic vacuum energy in (20) leads to a Planckian cosmological constant under the constraint (26) $[10,11]$. These problems can serve as pathfinders in constructing realistic QFTs.

The seventh important point is the sought SM-GR reconciliation: QFTs and the GR get unified as an intertwined whole

$$
S_{\mathrm{QFT} \cup \mathrm{GR}}=S_{\mathrm{QFT}}\left(g, \psi, \log \Lambda_{\wp}\right)+\int d^{4} x \sqrt{-g}\left\{-\frac{1}{2} M_{P l}^{2} R(g)-\frac{1}{4} c_{\phi} \phi^{\dagger} \phi R(g)-\frac{1}{16} c_{\varnothing} R(g)^{2}+\mathcal{O}\left(M_{P l}^{-2}\right)\right\}
$$

by way of gauge invariance in that gravity is incorporated into QFTs in a way restoring the gauge symmetries broken explicitly by their UV cutoff $\Lambda_{\wp}$. Gravity symmerges, that is, emerges for a symmetry reason [17, 10, 11]. Needless to say, $\delta \hat{S}, M_{P l}^{2}, c_{\phi}$ and 
$c_{\varnothing}$ are all bona fide quantum objects calculated in the flat spacetime such that their remnant $\log \Lambda_{\wp}$ dependencies can always be expressed in dimensional regularization via (22). They can be computed explicitly for a given QFT and the results can be tested via collider (like the FCC and dark matter searches), astrophysical (like neutron stars and black holes) or cosmological (like inflation and structure formation) phenomena. The action $S_{\mathrm{QFT} \cup \mathrm{GR}}$ is the master formula for all the cosmological, astrophysical and collider phenomena. Its field theory part (the SM plus a BSM sector) and gravity part (the GR plus higher-curvature scalar-tensor theory) are expected to evolve into wider and precise forms as progresses are made in collider experiments [29, 30], astrophysical observations $[31,22]$, and cosmological measurements [32, 22, 33].

\section{CONCLUSION}

The results speak for themselves. Quantum gauge invariance does indeed lead to a QFT-GR reconciliation. In the experimentallycompleted case of the SM, gravitational constant emerges rightly only if there exist new fields beyond the SM spectrum. These new fields, forming a BSM sector, can form dark matter, dark energy, and maybe more. The SM-GR reconciliation sets up a framework in which existing theoretical and experimental problems can be consistently addressed.

\section{ACKNOWLEDGEMENTS}

This work is supported in part by the TÜBİTAK grant 118F387. The author thanks H. Azri and İ. İ. Çimdiker for discussions.

\section{References}

[1] L. D. Landau and E. M. Lifshitz, Classical Theory of Fields, Pergamon Press, NY (1971).

[2] J. D. Norton, Rep. Prog. Phys. 56791458 (1993).

[3] E. Kretschmann, Annalen der Physik 48, 907 (1915); A. Einstein, Annalen Phys. 53, 575 (1917); R. D. Sorkin, Mod. Phys. Lett. A 17, 695 (2002).

[4] R. M. Wald, Einstein Stud. 14, 439 (2018) [arXiv:0907.0416 [gr-qc]].

[5] S. A. Fulling, Phys. Rev. D 7 (1973) 2850; V. Mashkevich, Notes on Quantum Field Theory in Curved Spacetime: Problems Relating to the Concept of Particles and Hamiltonian Formalism, arXiv:0706.1802v2 [physics.gen-ph].

[6] J. Mattingly, Einstein Stud. 11, 327 (2005); S. Carlip, Class. Quant. Grav. 25, 154010 (2008) [arXiv:0803.3456 [gr-qc]]; S. Boughn, Found. Phys. 39 (2009) 331 [arXiv:0809.4218 [gr-qc]].

[7] A. Ashtekar and A. Magnon, Proc. Roy. Soc. Lond. A 346, 375 (1975).

[8] E. P. Wigner, Annals of Mathematics 40, 149 (1939); V. Bargmann and E. P. Wigner, Proc. Natl. Acad. Sci. U.S.A. 34, 211 (1948).

[9] J. Polchinski, Nucl. Phys. B 231, 269 (1984).

[10] D. Demir, Naturalizing Gravity of the Quantum Fields, and the Hierarchy Problem, arXiv:1703.05733 [hep-ph].

[11] D. Demir, Adv. High Energy Phys. 2019, 4652048 (2019) [arXiv:1901.07244 [hep-ph]].

[12] M. E. Peskin and D. V. Schroeder, An Introduction to quantum field theory, Reading, USA: Addison-Wesley (1995); P. H. Chankowski, A. Lewandowski and K. A. Meissner, Acta Phys. Polon. B 48, 5 (2017) [arXiv:1608.01214 [hep-th]].

[13] J. Casas, A. Lleyda and C. Munoz, Nucl. Phys. B 471, 3-58 (1996) [arXiv:hep-ph/9507294 [hep-ph]].

[14] M. J. G. Veltman, Acta Phys. Polon. B 12 (1981) 437; G. F. Giudice, PoS EPS (2013) 163 [arXiv:1307.7879 [hep-ph]].

[15] Y. B. Zeldovich, JETP Lett. 6, 316 (1967); S. Weinberg, Rev. Mod. Phys. 61, 1 (1989).

[16] D. A. Demir, Found. Phys. 39, 1407-1425 (2009) [arXiv:0910.2730 [hep-th]]; Phys. Lett. B 701, 496 (2011) [arXiv:1102.2276 [hep-th]].

[17] D. A. Demir, Adv. High Energy Phys. 2016, 6727805 (2016) [arXiv:1605.00377 [hep-ph]].

[18] F. Bauer and D. A. Demir, Phys. Lett. B 665 (2008) 222 [arXiv:0803.2664 [hep-ph]]; C. N. Karahan, A. Altas and D. A. Demir, Gen. Rel. Grav. 45, 319 (2013) [arXiv:1110.5168 [gr-qc]]; H. Azri and D. Demir, Phys. Rev. D 95, 124007 (2017) [arXiv:1705.05822 [gr-qc]].

[19] V. Vitagliano, T. P. Sotiriou and S. Liberati, Annals Phys. 326, 1259 (2011) Erratum: [Annals Phys. 329, 186 (2013)] [arXiv:1008.0171 [gr-qc]]; D. Iosifidis, Class. Quant. Grav. 36, 085001 (2019) [arXiv:1812.04031 [gr-qc]].

[20] D. A. Demir, A Mechanism of Ultraviolet Naturalness, arXiv:1510.05570 [hep-ph].

[21] K. Hagiwara, S. Ishihara, R. Szalapski and D. Zeppenfeld, Phys. Rev. D 48, 2182 (1993); G. Cynolter and E. Lendvai, Cutoff Regularization Method in Gauge Theories, arXiv:1509.07407 [hep-ph].

[22] J. Beacham et al., J. Phys. G 47, 010501 (2020) [arXiv:1901.09966 [hep-ex]].

[23] K. Shimada, K. Aoki and K. i. Maeda, Phys. Rev. D 99, 104020 (2019) [arXiv:1812.03420 [gr-qc]].

[24] V. Faraoni, Phys. Rev. D 53, 6813 (1996) [astro-ph/9602111]; D. A. Demir, Phys. Lett. B 733 (2014) 237 [arXiv:1405.0300 [hep-ph]].

[25] R. Utiyama and B. S. DeWitt, J. Math. Phys. 3, 608 (1962); A. Salam and J. A. Strathdee, Phys. Rev. D 18, 4480 (1978).

[26] A. A. Starobinsky, Phys. Lett. 91B, 99 (1980).

[27] Y. Akrami et al. [Planck Collaboration], arXiv:1807.06211 [astro-ph.CO].

[28] K. Cankoçak, D. Demir, C. Karahan and S. Şen, Electroweak Stability and Discovery Luminosities for New Physics, [arXiv:2002.12262 [hep-ph]]; D. Demir and C. S. Un, Scalar Dark Matter and Electroweak Stability, [arXiv:2005.03589 [hep-ph]].

[29] S. Rappoccio, Rev. Phys. 4, 100027 (2019) [arXiv:1810.10579 [hep-ex]].

[30] A. Abada et al. [FCC], Eur. Phys. J. C 79, 474 (2019).

[31] A. Boveia and C. Doglioni, Ann. Rev. Nucl. Part. Sci. 68, 429 (2018) [arXiv:1810.12238 [hep-ex]].

[32] D. Rubin and J. Heitlauf, Is the expansion of the universe accelerating? All signs still point to yes a local dipole anisotropy cannot explain dark energy, arXiv:1912.02191 [astro-ph.CO].

[33] N. Arkani-Hamed and J. Maldacena, Cosmological Collider Physics, arXiv:1503.08043 [hep-th]. 